\title{
Le cheval au service de la communication et de l'efficacité des hommes dans les organisations
}

Hélène Dufau et Bernard Piazza

\section{(2) OpenEdition}

1 Journals

Édition électronique

URL : http://journals.openedition.org/communicationorganisation/2849

DOI : 10.4000/communicationorganisation.2849

ISSN : $1775-3546$

Éditeur

Presses universitaires de Bordeaux

Édition imprimée

Date de publication : 1 mai 2003

ISSN : 1168-5549

Référence électronique

Hélène Dufau et Bernard Piazza, «Le cheval au service de la communication et de l'efficacité des hommes dans les organisations », Communication et organisation [En ligne], 23 | 2003, mis en ligne le 27 mars 2012, consulté le 30 avril 2019. URL : http://journals.openedition.org/

communicationorganisation/2849 ; DOI : 10.4000/communicationorganisation.2849

Ce document a été généré automatiquement le 30 avril 2019

(c) Presses universitaires de Bordeaux 


\title{
Le cheval au service de la communication et de l'efficacité des hommes dans les organisations
}

\author{
Hélène Dufau et Bernard Piazza
}

1 Il y a quelques mois, un encadré publié dans Courrier International faisait état d'une dimension particulière de la formation à l'intérieur de la Manchester Business School qui pouvait apparaître insolite, voire inconcevable pour certains. Il s'agissait d'effectuer une partie de la formation en management avec la contribution de chevaux. De fait, pour quiconque connaît les grands traités d'équitation des siècles passés, rien de particulièrement étrange à cette démarche, même si l'évolution technique explique qu'elle soit tombée dans l'oubli au fil des siècles. Voyons comment, au xxe siècle, cette pratique peut répondre à des besoins existant dans les organisations.

2 Parmi toutes les difficultés auxquelles se trouvent confrontées les organisations dans un monde hautement instable et évolutif, l'une d'entre elles, récurrente et fondamentale, réside dans la sélection, le recrutement et l'organisation du travail de leurs agents. Comment déceler dans la pléthore de candidats disponibles, tous dotés par ailleurs de niveaux de formation et de technicité suffisants, la personne qui se trouvera en adéquation idéale avec les fonctions à remplir, les responsabilités à prendre, les savoirfaire et les savoir-être à mettre en œuvre, tout en s'adaptant souplement au milieu et à la culture en place, faite d'habitudes, de personnalités multiples, de contraintes humaines et environnementales diverses, etc.. Cette quête, qui peut paraître relever d'une gageure, constitue pourtant l'une des obligations permanentes des organisations, confrontées à des mouvements divers de personnels, à leur évolution propre, à celle de l'environnement concurrentiel ou partenarial. Ces changements perpétuels les contraignent à s'adapter sans cesse afin d'assurer leur pérennité, ainsi que celle des fonctions quelles remplissent, que ces dernières soient de production de biens ou de services. Les contraintes de l'environnement économique, à travers l'intensification de la mondialisation, deviennent de plus en plus fortes, et renforcent encore le défi que 
représente l'identification, le recrutement et l'employabilité des agents dont l'organisation doit s'assurer les services.

3 Si les organisations administratives ${ }^{1}$ ont résolu cette difficulté d'une manière standardisée, en organisant des concours, il n'en va pas de même pour l'organisation entrepreneuriale pour laquelle cette course à l'oiseau rare représente l'un des défis les plus ardus à résoudre. Il faut de surcroît agir avec le moins d'échecs possibles, car ces derniers sont coûteux en temps, en énergie, et en argent.

Une fois la personne recrutée, la question n'est pas pour autant résolue. Il s'agira cette fois de s'assurer d'une participation efficiente de l'agent au fonctionnement de l'organisation, et de son implication suffisante dans ses intérêts. Cette participation n'est pas chose simple, car outre la mise en œuvre de compétences techniques, elle implique dans le cas des agents d'encadrement et de direction, de fonctions de gestion de ressources humaines, lesquelles constituent par elles-mêmes un défi fondamental pour le fonctionnement de l'organisation, mais aussi pour les individus. À ce stade, la recherche est celle de personnes capables de mobiliser, de motiver, de guider leurs collaborateurs vers la réalisation d'un but commun. En un mot, celle d'individus dotés de leadership. Là encore, une multitude de techniques ont été employées pour tenter de vérifier les aptitudes ou de susciter les attitudes dont l'organisation a besoin: la capacité de commandement, d'entraînement, la dynamique d'un groupe, la confiance réciproque de ses membres, leur solidarité, etc. On se rappellera à cet égard la floraison des « séminaires outdoor", et autres stages de motivation, d'intégration, et leur modalités variées, lesquelles, là aussi, peuvent susciter étonnement ou interrogation (sauts à l'élastique, etc.).

5 Or, pour qui connaît les relations entre l'homme et le cheval, il y a finalement une dimension très logique dans l'emploi de ces dernières pour cerner certaines des qualités de l'homme que l'on va faire agir en milieu de travail, et pour déceler certaines attitudes intérieures, pour lesquelles le cheval pourra servir de détecteur "sincère " et non ambigu, pour peu que l'on exploite certaines de ses caractéristiques comportementales. En effet, le cheval est une proie, dont l'une des préoccupations majeures, outre celle de se nourrir, est de fuir tout ce qui peut représenter un danger pour lui. Pour reprendre Pat Parelli², le cheval recherche essentiellement la sécurité, le confort, le jeu. Son caractère grégaire n'en fait pas un fuyard solitaire, mais bien au contraire un être sociable qui compte sur le groupe et sur ses leaders pour assurer sa sauvegarde. Ainsi, il se rapprochera naturellement de celui qui le rassure, par exemple l'étalon chargé de veiller sur la sécurité de tous, et la jument dominante, qui possède une fonction régulatrice et éducative au sein du groupe. En outre, au sein des hardes de chevaux, s'élaborent et se négocient en permanence des relations hiérarchiques entre les individus, lesquelles se traduisent selon les circonstances par des préséances pour accéder aux ressources (eau. facilités relationnelles, voire accouplements) l'étalon responsable du groupe bénéficiant évidemment de la totalité des avantages. Dans la même logique, le cheval va se rapprocher d'un humain si celui-ci manifeste, par son attitude et ses comportements, des qualités de leadership, ou se montrer dominateur face à un humain qui doute de la légitimité de l'exercice de son autorité. Il va également fuir un "dictateur», ou douter d'un instable.

6 En outre, le mental «nature » du cheval lui confère une sincérité de réaction sur laquelle on peut également compter. Pour lui, pas de dissimulation, de réactions ambiguës, ou calculées, pas d'arrière-pensées. Pas de « oui, mais », de " peut-être » ou d'à peu près. Et 
donc pas d'interprétations difficiles à démêler. À cet égard, la relation avec lui présente un avantage sur d'autres pratiques d'évaluation, ou sur l'interaction avec des humains. Car si des techniques d'évaluation comme la mise en situation au cours d'activités stressantes permettent de faire réagir les agents qui en sont la cible, elles ne renvoient finalement l'individu qui s'y trouve confronté qu'à lui-même, dans un effet miroir où le sujet est également objet. S'il y a interaction avec d'autres humains, c'est une difficulté supplémentaire, car l'interaction construit, mais brouille les repères et ne permet plus de discerner ce qui appartient aux uns et aux autres. Avec le cheval dont le comportement, relativement bien cerné grâce aux données éthologiques dont on dispose, est finalement prédictible, ces biais sont contournés. Il s'agit là d'un partenaire non-humain mais interprétable, susceptible de réactions autonomes et motivées qui place le sujet face à luimême dans une interaction simplifiée mais significative.

Cette argumentation et ce qui va suivre s'appuie sur l'expérience de Bernard Piazza, Moniteur d'équitation depuis une dizaine d'années dans la région bordelaise ${ }^{3}$, pratiquant et formateur en équitation éthologique. et qui propose une lecture explicative des mécanismes à l'œuvre, notamment dans la démarche de formation de la Manchester Business School (mais également dans d'autres, comparables dans leur approche et leurs mécanismes).

\section{Le Leadership}

Pour notre interlocuteur, un leader humain susceptible de convaincre le cheval est une personne capable de maîtriser son mental, ses réactions émotionnelles, et ses comportements physiques, lorsqu'elle se trouve en position d'autorité (chercher à obtenir quelque chose d'un partenaire), et ce aussi bien dans le cadre de l'équitation que dans celui de l'organisation. La position d'autorité génère souvent, même inconsciemment, une peur de l'échec et une insécurité intérieure qui se dépasse plus ou moins aisément. Aussi, atteindre un état efficace de leadership se traduit-il par : un dégagement d'énergie physique (couplé ou non à des mouvements),

10 la capacité à mettre en place des stratégies, de les adapter ou d'en changer en cas de besoin, la capacité de fragmenter ses objectifs et d'auto-évaluer son action, la persévérance dans l'acte commencé, le contrôle d'émotions négatives comme la peur, la frustration, la colère, le contrôle des projections et suppositions négatives comme le découragement ( je n’y arriverai jamais »), la tentation d'abandonner la partie.

14 et au contraire, la capacité de positiver qui, avec vigilance, amène au « dépassement de soi ».

15 Les démonstrations de certains élèves de Pat Parelli le montrent ${ }^{4}$, il ne s'agit pas de mettre en œuvre des capacités physiques comme être jeune, ou fort, ou courir vite. Il s'agit plutôt de mobiliser et de dégager une énergie suffisante, tout en restant relaxé mais réactif. Le leadership se fonde par conséquent sur une intégration harmonieuse des réactions physiques et du contrôle émotionnel et mental.

16 C'est pourquoi des activités équestres, ou simplement des contacts modulés avec le cheval (qui recherche par nature le leadership) en fonction de chaque personne, permettent de mettre en évidence les qualités de confiance et de maîtrise de soi des individus, leur 
capacité de maîtrise mentale et émotionnelle, voire leurs dispositions inconscientes d'agressivité, de bienveillance ou de mollesse... Il ne s'agit pas là de spéculations hasardeuses : faire appel à la dimension cognitive du processus permet d'en éclaircir les mécanismes. De fait, ce n'est plus la partie réflexive et intellectuelle de l'humain qui se trouve mise à contribution dans ce processus, mais la partie émotionnelle et affective de l'individu (dimensions du plaisir, de l'angoisse, de la colère, de l'amour et de la haine), gérée par le cerveau limbique, avec laquelle le cheval entre en résonance. Et celui-ci est à cet égard un partenaire expert à lire les émotions à travers les plus petits comportements sensori-moteurs de ses vis-à-vis, c'est-à-dire les mouvements corporels ou de physionomie. Le «travail » peut être révélateur, qu'il soit à pied, en longe, monté, ou en liberté, avec un ou plusieurs équins ${ }^{5}$

\section{Le contrôle du mental}

17 Contrôler son mental, dans ce contexte, cela veut dire être capable à la fois d'employer à bon escient ses connaissances sur l'homme et les situations, mais également l'état d'esprit dans lequel on le fait. Ce sera par exemple ne pas agir en prédateur qui cherche à « coincer » son partenaire, ne pas agir pour la galerie, ne pas chercher le succès, c'est-àdire être modeste, humble (mais pas humilié) ou, comme le dit Pat Parelli, être «naturel, progressif, et positif ». En fait, il s'agira d'exercer sa capacité d'auto-évaluation en toute tranquillité, ne pas prendre mais demander, puis s'il le faut, dire et promettre, c'est-àdire affirmer sa demande et ne laisser aucun doute sur sa détermination à l'obtenir ou « faire preuve de " persévérance polie dans l'attitude convenable » (P. Parrelli). Et ceci en restant toujours empreint d'une détermination douce et bienveillante.

Bien sûr. on peut se demander selon quelle méthode appliquer la relation avec les chevaux pour mesurer ces dimensions de l'individu. En pratique, on peut élaborer des processus proportionnés à l'expérience équestre des personnes, ou à la crainte inspirée par l'animal. Si par exemple quelqu'un a très peur des chevaux, ce qui se conçoit lorsqu'il n'y a aucune expérience dans ce domaine, on peut simplement lui demander de traverser un paddock où des animaux broutent tranquillement, ou d'y stationner un moment (ce qui pourra susciter la curiosité des chevaux, et générer une réaction de leur part). On pourra de ce fait mesurer la capacité qu'il ou elle a de surmonter le trouble occasionné par l'entrée en milieu inconnu ou hostile. Cette circonstance n'est pas étrangère à celle que peut rencontrer un chef de service ou un dirigeant qui doit entrer dans un bureau ou un atelier et faire face à l'hostilité de ses subordonnés ou collaborateurs. On pourra ensuite lui demander d'approcher l'animal, de le caresser, de lui mettre un licol, le faire sortir, le mettre en mouvement, etc. Il aura là autant d'éléments susceptibles d'interprétation: de quelle manière la personne va-t-elle surmonter sa difficulté ? Va-telle aller directement à la tête de l'animal, le regarder en face, s'approcher de manière assurée ou hésitante ? Ses autres mouvements seront-ils également assurés ou hésitants? Va-t-elle bondir au moindre mouvement, se hasarder à caresser l'animal, le corps ? la tête? Va-t-elle s'enhardir rapidement, faire assez vite preuve de confiance en elle, ou rester assez longtemps inhibée par ses propres craintes? Va-t-elle parler ou non. avec quel à propos, avec quelle intensité et à quel rythme ? Ses paroles seront-elles en accord avec le langage de son corps? Ce même test simple

19 (rentrer dans un paddock et approcher voire toucher un équin) peut s'adresser et avoir valeur quel que soit le niveau équestre préalable du sujet. On peut jouer sur des 
paramètres comme le nombre d'animaux, leur âge, la cohésion du groupe, le temps séparant celui-ci du repos, la place sociale de l'animal-cible (on risque de voir l'interposition du dominant entre lui et l'humain), la taille et la forme de l'espace dans lequel se déroule l'exercice, les événements qui se produisent en dehors du paddock (bruits, mouvements, présence d'humains ou d'autres animaux, nourriture), le degré de confiance du ou des animaux dans l'humain en général, etc. Ainsi, une palette très riche s'offre à celui qui gère la situation pour permettre d'affiner les évaluations. On peut quasiment moduler à l'infini les situations et reporter ces variations sur une multitude d'autres tests éventuels, et toujours quel que soit le niveau équestre des sujets testés.

Il est très facile pour le professionnel expérimenté (mais aussi pour le cavalier attentif à ses états émotionnels), d'évaluer les réactions des personnes placées en situation, comme de générer des situations inattendues ${ }^{6}$ qui peuvent permettent de mettre en œuvre des difficultés significatives pour les personnes, rassurer ou déstabiliser, intervenir pour simplifier ou compliquer selon les besoins ou l'évolution de la situation. On peut donc organiser des activités expérimentales, fournir une aide ou non, donner des consignes, et observer le comportement de la personne. Est-elle sous la coupe de ses émotions, ou capable de dépasser ces dernières pour entendre, comprendre, et appliquer les consignes reçues, et percevoir les signaux émis par son ou ses partenaires? Est-elle capable de se comporter " avec intelligence ", au sens de s'adapter à des nouveautés (même si elles sont provoquées par l'animateur) ? Pour un professionnel habitué à la formation équestre, et éclairé sur la communication et les réactions humaines, tous ces comportements sont significatifs et susceptibles d'éclairer la personne en question.

Une autre dimension peut également consister à lui soumettre des questions d'évaluation sur ce qu'elle a pu faire (à pied ou à cheval, selon les cas) : fait-elle preuve d'un excès d'autosatisfaction (générée par le sentiment

subjectif d'une "réussite de survie ». quelque soit la demande, et pas par la satisfaction liée à une évaluation plus objective de sa réussite face à l'exercice demandé). Fait-elle preuve d'auto-dénigrement? Une personne peut paraitre forte, mais ne l'être qu'avec les êtres qu'elle perçoit comme plus faibles qu'elle... Ce sont là des caractéristiques que l'on peut avoir besoin de rechercher et de mesurer, et dans ce cas. le cheval devient sans difficulté une «mesure de justesse». et ce quels que soient les tests que l'on effectue, à pied ou monté. Dans le cas évoqué ci-dessus, même à pied, si les circonstances correspondantes sont créées, l'animal va éluder le contact et éviter la personne. Ce type de «mesure de justesse » par le cheval a pour avantage qu'il ne comporte pas d'arrièrepensée, de calcul, de prévision, pas de recherche de gloire ou d'estime. L'animal ne sera pas influencé par le grade, les diplômes, l'intelligence, la réussite professionnelle ou le statut social de la personne en question, ni par ses vêtements ou tout autre élément de prestige. En outre, le cheval n'est pas soumis à un chef de meute mais, soucieux de sa sécurité et de son bien-être, il sait évaluer les situations qui le menacent et s'il est maltraité ou inquiet, fuit ou se rebelle, quelle que soit l'occasion.

On retrouve ici une relation tripartite (entre la personne en situation, le cheval - ou le groupe de chevaux -, le milieu matériel et humain, le site et l'animateur) qui mettent en œuvre, ainsi que l'ont défini Mucchielli. Corbalan et Ferrandez ${ }^{7}$. les processus de contextualisation spatial, physique et sensoriel, du positionnement et de structuration des relations, du temps. 


\section{Le contrôle émotionnel} telles que la peur, la colère, la frustration, qui surgissent parce qu'on ne réussit pas ce que l'on vient d'essayer (et d'autant plus facilement qu'on est confronté à un partenaire vivant, sincère et réactionnel). On est là dans un domaine de communication non-verbale, ce qui va permettre de placer les individus dans une relation significative et immédiatement réactive avec un "expert en la matière " qui ne passera rien si la situation est bien menée. Bien que les exercices à effectuer avec un ou des chevaux puissent être simples et non dangereux, ils sont significatifs car

le partenaire est un être vivant, extrêmement sensible, on l'a vu, aux micromouvements, aux attitudes corporelles, à ce qui s'affiche sur le visage, aux mimiques, car ce sont ces éléments qui lui servent naturellement à gérer ses relations sociales dans le groupe. Cette situation est inhabituelle pour beaucoup de personnes, dont la formation académique met lourdement l'accent sur les fonctions de raisonnement ou de mémorisation tout en négligeant, en règle générale, les situations de communication autres que la parole et surtout l'écrit. On peut ainsi voir émerger des réactions interprétables par qui connaît l'éthologie de l'animal... et humaine.

On va pouvoir vérifier l'émergence d'émotions négatives comme le découragement, la tentation d'abandonner, qui apparaît plus ou moins rapidement face à la difficulté, ou l'apparition de projections et de suppositions négatives, du type "je n'y arriverai jamais", "c'est trop dur pour moi", "les autres sont capables mais pas moi", ou une réaction d'agressivité qui se manifeste au plan gestuel avec plus ou moins de netteté contre ce qui ne se soumet pas.

Ainsi, loin d'être une démarche farfelue et arbitraire, l'évaluation du comportement émotif et relationnel face à des chevaux peut constituer une démarche qui mette en œuvre de manière pragmatique ce que l'on sait du fonctionnement cérébral en termes de comportements émotionnel et affectif, lesquels ont toujours une incidence sur les capacités et comportements relationnels de l'homme au travail. Sans vouloir à tout prix que cette démarche s'amplifie et se répande, on peut comprendre son introduction même temporaire - dans le cursus de la Manchester Business School, pour laquelle il serait sans doute intéressant de mesurer la pertinence, l'impact et le devenir après application à la formation de quelques promotions de managers.

De la même façon que pour commander ou conclure un contrat, le leader est celui qui fait en sorte que son idée devienne celle de son vis-à-vis, tout en laissant croire à ce dernier qu'il a de bonnes idées, et que c'est celles-ci que l'on suit. Ces tests sont pratiqués quotidiennement dans notre centre, non dans un but de sanctionner ou de recrutement, mais pour éduquer (et parfois rééduquer) les enfants ou les adultes qui, grâce et par le cheval ou le poney, peuvent apprendre sur eux, même sans en avoir conscience.

Les plus efficaces restent ceux qui sont :

- capables d'être le plus doux possible et, s'il le faut, aussi fermes que nécessaire capables d'agir ici et maintenant

- capables de se situer par rapport au cheval à des niveaux différents (c'est-à-dire en adaptant sa psychologie et son comportement à celui de leur vis-à-vis. c'est-à-dire en « se pensant et en agissant cheval »)

Communication et organisation, 23 | 2003 
- capables de mettre en œuvre des qualités d'observation et d'analyse des comportements d'autrui. puis d'eux-mêmes. communication en milieu professionnel, qu'il s'agisse de formation, d'encadrement, de motivation, et qui ne sont peut-être, au fond, que l'équivalent de ce que l'on appelle diplomatie, respect, maîtrise de soi. sagesse, bienveillance... toutes qualités qu'il est si difficile d'enseigner.

31 À cet égard, une formation équestre bien menée constitue une magnifique démarche d'apprentissage.

\section{NOTES}

1. On peut se demander si le système des eoneours. malgré ses qualités « d'objectivité ». ne met pas finalement l'accent sur des dimensions trop particulières des recrutés, notamment la capacité à « bûcher ». et à résister à des stress « passifs ». sans tenir suffisamment compte d'une adaptation psychologique et mentale à la fonction envisagée. Qu'il s'agisse d'être magistrat, enseignant, chef de service.... le candidat sera peut-être manipulable. ou rigide, faute de pouvoir assumer les dimensions d'autorité et de leadership inhérentes à la fonction qu'il occupe.

2. Pat Parelli a développé une méthode d'éducation éthologique. "naturelle ». pour les chevaux. Saturai Horsemanship.qu'il enseigne depuis plus de 20 ans dans le monde entier. Voir le site www.parelli.com

3. Bernard Piazza anime le club Trotteland. 94 avenue St Jacques de Compostelle. 33610 - Cestas. tél. 0556788008

4. Silke. une des « élèves de Pat Parelli ». paralytique, effectue des démonstrations édifiantes de ce phénomène en fauteuil roulant

5. On notera au passage la cohérence de ces propositions avec les doctrines des arts martiaux ${ }^{5}$ qui évoquent les dimensions de l'énergie vitale et de sa nécessaire maîtrise telle qu'elle se manifeste à travers l'usage $\mathrm{du}$ « $\mathrm{ki}$ » dans diverses pratiques

6. On supposera bien sûr pour ce genre d'expérience que l'on dispose d'une cavalerie fiable, bien dressée, non stressée, de caractère stable et paisible, car il ne s'agit pas de mettre les personnes en danger, mais bien de générer une situation significative en compagnie d'un être sensible et réactif, et ce avec le maximum de sécurité.

7. In: Alex Mucchielli. J.-A. Corbalan et Valérie Ferrandez. Théorie des processus de la communication. Armand Colin. 1998. 177 p. 


\section{RÉSUMÉS}

Mettre quelqu'un en interaction avec des chevaux c'est. pour qui connaît les modalités du comportement éthologique et de communication de ce dernier, se donner le moyen de constater chez son partenaire humain un ensemble de réactions qui ont trait à la maitrise des émotions, du mental, les qualités de leadership. Les organisations peuvent tirer parti de ces possibilités pour identifier le potentiel et les blocages de communication qui peuvent affecter leurs membres et. partant, leur fonctionnement et l'efficacité des collaborations en leur sein.

Interaction with Horses, for people who know how they behave ethologically, and how they communicate, may he a way of identifying, in the inleracting human partner, a set of éléments which inform about the mental, emotional. and leadership eharacteristics of the persan. Organizations may use thèse possihilities to identity the communication capacities and limits of its memhers. and therefore how they affect their collaborative effciency.

\section{INDEX}

Mots-clés : communication homme/animal, communication non-verbale, cheval, management, leadership

\section{AUTEURS}

\section{HÉLÈNE DUFAU}

Hélène Dufau est maître de Conférences à l'Université Bordeaux I où elle enseigne la communication. Ses recherches portent sur les mécanismes de la communication nonverbale, et les conditions de l'empathie, observées notamment à travers les contacts avec les animaux, et en particulier la pratique de l'équitation éthologique.

\section{BERNARD PIAZZA}

Bernard Piazza, titulaire d'une maîtrise en droit, est éducateur en équitation sportif titulaire du Brevet d'État, et éleveur de chevaux et poneys. Il anime depuis une dizaine d'années un club d'équitation dans les environs de Bordeaux, à Cestas. Il pratique depuis plusieurs années l'équitation éthologique (Parelli Natural Horsemanship - niveau 2). 11 s'est aussi formé à la méthode développée par Elisabeth de Corbigny (Rec Farm, Route de Bastonies. 09500 Mirepoix). Il assure également un enseignement d'équitation éthologique. du débutant au cavalier confirmé. 